\title{
Sixth Asia-Pacific EPR/ESR Symposium (APES 2008) Cairns Australia 13-18 July, 2008
}

\author{
Michael J. Davies • Graeme R. Hanson • John R. Pilbrow
}

Published online: 20 October 2009

(C) Springer 2009

APES 2008 was held in the city of Cairns, the gateway to far north Queensland, Australia. This was the sixth in the series that has taken place since 1997 (Hong Kong) and subsequently in 1999 (Hangzhou), 2001 (Kobe), 2004 (Bangalore) and 2006 (Novosibirsk). The APES meetings bring together scientists from nations on the Pacific rim, particularly from China, Hong Kong, Japan, South Korea, India, Vietnam, Russia, Australia and New Zealand and also from other parts of the world including the USA, Europe and Africa.

The Cairns Convention Center located in the tropical paradise of Cairns was chosen as the venue for APES 2008 for its world-class facilities and professional team which helped enormously in the organization of the meeting. Cairns is a vibrant tropical city fringed by golden sandy beaches, the Great Barrier Reef and World Heritage Rainforest. The delegates who attended the meeting would no doubt attest that Cairns has the best of everything-great restaurants, excellent accommodation, exciting shopping, a casino and abundance of cruises, tours and attractions, complemented by a lively cosmopolitan atmosphere.

The scientific program began on Sunday with a workshop entitled "Workshop on Multifrequency EPR: Applications to Complex Systems" which was designed to provide a series of introductory lectures into the application of EPR in diverse areas from physics through chemistry to biology and medicine. Following the workshop,

\footnotetext{
M. J. Davies

Heart Research Institute, 7 Eliza St., Newtown, Sydney, NSW 2042, Australia

e-mail: daviesm@hri.org.au

G. R. Hanson $(\bowtie)$

Centre for Magnetic Resonance, The University of Queensland, Brisbane, QLD 4072, Australia e-mail: Graeme.Hanson@cmr.uq.edu.au 
the scientific program consisting of oral and poster sessions addressed the application of high-resolution EPR spectroscopy and imaging to quantum computing, advanced materials, photosynthesis, biological inorganic chemistry, inorganic and organic chemistry and medicine.

The President of the Asia-Pacific EPR Society, Prof. Hitoshi Ohta (2004-2008) was honored by the International EPR Society through the award of a Silver Medal in Instrumentation and delivered a lecture entitled "Direct Determination of the Spin Gap in the Quantum Spin System by High-Frequency ESR".

The inaugural Young Scientist's Awards of the Asia-Pacific EPR Society were instituted to recognize outstanding early career scientists in the Asia-Pacific region who undertake research in EPR spectroscopy. The 2008 award was presented jointly to Dr. Simon Drew (Non-coincidence with MoV-dithiolate Folding AngleRelevance to Molybdenum Enzyme Structure and Function) and Dr. Takanari Kashiwagi (Spin Excitations in the Field-Induced Phase of the Quasi OneDimensional $S=1$ Heisenberg Antiferromagnet NDMAP) who have undertaken research in biological inorganic chemistry and advanced materials, respectively. Both recipients gave excellent and lucid lectures describing their results that have been published in internationally recognized peer-reviewed journals.

Poster prizes were awarded to Sophia Goodchild (Macquarie University, Australia) for her poster entitled "SDSL-EPR Studies of the CLIC Chloride Ion Channel Family" and to Kenta Kondo (Kobe University, Japan) for his poster entitled "Development of a High-Field ESR System Using He-Free Superconducting Magnet".

Annual General Meetings of the Asia-Pacific EPR Society and the International EPR Society were held during the Symposium ensuring the continued development of EPR spectroscopy and its application in the Asia-Pacific region and throughout the world.

We would especially like to thank our sponsors (Jeol, Bruker BioSpin, Cryogenics and Springer) for their financial support of the conference that enabled the provision of Travel Bursaries to scientists in the Asia-Pacific region to enable them to attend this meeting. 